\title{
Nonlinear Flatness-Based Controller for Permanent Magnet-Excited Synchronous Motor
}

\author{
Pham Tam Thanh ${ }^{a}$ and Nguyen D. That ${ }^{a, b}$ \\ ${ }^{a}$ Faculty of Electrical and Electronics Engineering, Vietnam Maritime University, Vietnam \\ ${ }^{b}$ Faculty of Engineering and Information Technology, Sydney, NSW 2007, Australia \\ E-mail:phamtamthanh@vimaru.vn, That.NguyenDinh@uts.edu.au
}

\begin{abstract}
:
This paper addresses the problem of nonlinear discrete-time flatness-based controller design for a Permanent Magnet-Excited Synchronous Motor (PMSM). To eliminate the static errors of the system state variables and consider the nonlinear characteristics of a PMSM, a cascaded flatnessbased control scheme is proposed. Simulation results are provided to illustrate the effectiveness of the proposed control structures, in terms of better performance.
\end{abstract}

Keywords:

Flatness-based control; Permanent Magnet-Excited Synchronous Motor (PMSM); nonlinear control; real-time control.

\section{NOMENCLATURE}

\begin{tabular}{|c|c|c|}
\hline Symbol & Unit & Description \\
\hline $\mathbf{A}, \mathbf{B}, \mathbf{N}, \mathbf{S}$ & & Matrices of model \\
\hline f & & Nonlinear function \\
\hline$L_{s}, L_{r}$ & $\mathrm{H}$ & Stator, rotor inductance \\
\hline$L_{m}$ & $\mathrm{H}$ & Mutual inductance \\
\hline$R_{s}, R_{r}$ & $\Omega$ & Stator, rotor resistance \\
\hline$u_{s d}, u_{s q}$ & $\mathrm{~V}$ & $d q$ components stator voltage \\
\hline$i_{s d}, i_{s q}$ & A & $\begin{array}{l}d q \text { components of stator } \\
\text { current }\end{array}$ \\
\hline$T_{\mathrm{s}}, T_{\mathrm{r}}$ & S & Stator, rotor time constants \\
\hline$L_{s d}, L_{s q}$ & $\mathrm{H}$ & $d$ axis, $q$ axis inductance \\
\hline$\omega_{s}, \omega$ & $\mathrm{rad} / \mathrm{s}$ & $\begin{array}{l}\text { Stator circuit velocity, } \\
\text { Mechanical rotor velocity }\end{array}$ \\
\hline$\vartheta_{s}$ & $\mathrm{rad}$ & $\begin{array}{l}\text { Angle of flux orientated } \\
\text { coordinate system }\end{array}$ \\
\hline$\sigma$ & & Total leakage factor \\
\hline$\psi_{p}$ & $\mathrm{~Wb}$ & Pole flux \\
\hline$J$ & $\mathrm{~kg} \mathrm{~m}^{2}$ & Torque of inertia \\
\hline$m_{W}$ & $\mathrm{Nm}$ & Load torque \\
\hline$z_{p}$ & & Number of pole pairs \\
\hline
\end{tabular}

\section{Introduction}

With the advantages of superior power density, high performance motion control with fast speed and enhanced accuracy, Permanent Magnet-Excited Synchronous Motors (PMSM) have been increasingly used in robotics, precision machining and many automation processes. Therefore, the problem of control design for PMSM has received considerable attention. However, there remain interesting questions as to how to design a controller so that the static errors of the system state variables are minimized in which the nonlinear characteristics of the PMSM are taken into account.

The concept of differentially flat nonlinear systems was first introduced by Fliess et al, 1992. The system is considered to be flat if a set of outputs can be found such that all states and inputs can be determined from these outputs without integration. The main purpose of the flatness-based control method is first to design an open-loop nominal control corresponding to the predicted trajectory of the flat output. Then, a feedback control law is applied to stabilize the real trajectory around the predicted trajectory of the flat output. The flatness-based control has been recognized as a promising method to deal with nonlinear systems (see, e.g., Levine, 2009 and references therein). Based on the combination of the natural energy dissipation properties of the permanent magnet stepper motor system with its differential flatness property, a nonlinear feedback controller was proposed in Ramirez, 2000. To minimize the copper loss at all operating points of the PMSM, a hierarchical flatness-based control scheme was developed in Delaleau et al. 2004. In Dannehl and Fuchs 2006, a nonlinear differential flatness-based control was proposed to the induction machine fed by a voltage source converter in which the flatness-based control was used for the inner current and outer flux and speed loops. Another approach for drive systems with elastically coupled loads was reported by Thomsen and Fuchs 2010. By using fuzzy logic technique to eliminate the effects of the time-varying nonlinearities of an induction 
motor, a fuzzy differential flatness-based controller was developed by Fan and Zhang, 2011. In Houari et al. 2012, a new differential flatness-based control method was presented for a three-phase inverter with an $L C$ filter. Recently, quasi-continuous implementation of structural nonlinear controller based on directdecoupling for PMSM was reported in Thanh and Quang, 2013.

It should be noted that in the aforementioned papers, the problem of nonlinear flatness-based control has not been fully investigated and the minimization problem of the system state variable static errors has not received considerable attention. These facts have been motivated us to the present study.

In this paper, the problem of nonlinear discrete-time flatness-based controller design for PMSM is investigated. By considering the nonlinear characteristics of PMSM, a control strategy based on flatness theory is proposed to suppress the system state static errors. Simulation results are given to illustrate the effectiveness of the proposed approach.

The paper is organized as follows. Section 2 presents a nonlinear flatness-based controller design method for PMSM. Simulation results for a standard PMSM are given in Section 3. Section 4 concludes the paper.

\section{Nonlinear flatness-based controller design for PMSM}

\section{$2.1 \quad$ Flatness-based control}

As mentioned in Fliess et al. 1994 and Fliess et al. 1995, the main property of differential flatness is that the state and input variables can be directly expressed, without integrating any differential equation, in terms of the flat output and a finite number of its derivatives. Therefore, the trajectory of input can be determined from desired trajectory of flat output. The general flatness-based control structure consists of a nominal feedforward controller combined with a feedback stabilizing controller as shown in Figure 1.

In this structure, the feedback controller is crucial of importance to compensate the effects of external disturbances and model uncertainties.

\subsection{Flatness-based controller for PMSM}

To eliminate the system state static errors and consider the nonlinear characteristics of PMSM, a cascade nonlinear flatness-based control structure is proposed in Figure 2.

As shown in Figure 2, the cascade control structure includes two loopss which are coupled to each other.
The outer loop (speed loop) consists of a proportinalintegral controller (PI-controller) and a current Feedforward block while the inner loop (current loop) containing another PI block combined with a voltage Feedforward block. Here, the current controller for the current loop is first designed to guarantee that $i_{s d} \rightarrow i_{s d}^{*}, i_{s q} \rightarrow i_{s q}^{*}$ sufficiently fast with respect to the variations of the desired trajectories $\omega \rightarrow \omega^{*}$ which will be achieved by the mechanical subsystem (speed loop). Then, a speed controller is synthesized. It should be noted that these PI blocks are used to compensate the current and speed static errors.

\subsubsection{Stator current controller}

Motivated by Quang and Dittrich, 2008, we consider the continuous-time model current of PMSM as follows

$\left\{\begin{array}{l}\dot{x}=f(x)+H(x) u=f(x)+h_{1}(x) u_{1}+h_{2}(x) u_{2}+h_{3}(x) u_{3}, \\ y=g(x),\end{array}\right.$
where

- State vector:

$x=\left[\begin{array}{lll}x_{1} & x_{2} & x_{3}\end{array}\right]^{T}=\left[\begin{array}{lll}i_{s d} & i_{s q} & \vartheta_{s}\end{array}\right]^{T}$,

- Input vector:

$u=\left[\begin{array}{lll}u_{1} & u_{2} & u_{3}\end{array}\right]^{T}=\left[\begin{array}{lll}u_{s d} & u_{s q} & \omega_{s}\end{array}\right]^{T}$,

- Output vector:

$y=\left[\begin{array}{lll}y_{1} & y_{2} & y_{3}\end{array}\right]^{T}=\left[\begin{array}{lll}x_{1} & x_{2} & x_{3}\end{array}\right]^{T}$,

$f(x)=\left[\begin{array}{lll}-c_{1} x_{1} & -d_{1} x_{2} & 0\end{array}\right]^{T}$,

$H(x)=\left[\begin{array}{lll}h_{1}(x) & h_{2}(x) & h_{3}(x)\end{array}\right]$,

$h_{1}(x)=\left[\begin{array}{lll}a_{1} & 0 & 0\end{array}\right]^{T} ; h_{2}(x)=\left[\begin{array}{lll}0 & b_{1} & 0\end{array}\right]^{T}$,

$h_{3}(x)=\left[\begin{array}{lll}\frac{a_{1}}{b_{1}} x_{2} & -\frac{b_{1}}{a_{1}} x_{1}-b_{1} \psi_{p} & 1\end{array}\right]^{T}$,

$g(x)=\left[\begin{array}{lll}g_{1}(x) & g_{2}(x) & g_{3}(x)\end{array}\right]^{T}=\left[\begin{array}{lll}x_{1} & x_{2} & x_{3}\end{array}\right]^{T}$,

and temporary parameters:

$a=\frac{1}{L_{s d}} ; b=\frac{1}{L_{s q}} ; c=\frac{1}{T_{s d}} ; d=\frac{1}{T_{s q}}$.

Note that the functions $f($.) and $H($.) in equation (1) are nonlinear in nature, the ordinary differential equation (1) cannot be solved exactly, and hence the exact form of the discrete-time differential equation is difficult to obtain. Therefore, to obtain the discrete-time current model of PMSM, Taylor's series expansion is used

$x(k+1)=x(k)+\left.\dot{x}(t)\right|_{t=k T} T+\Xi(T)$, 


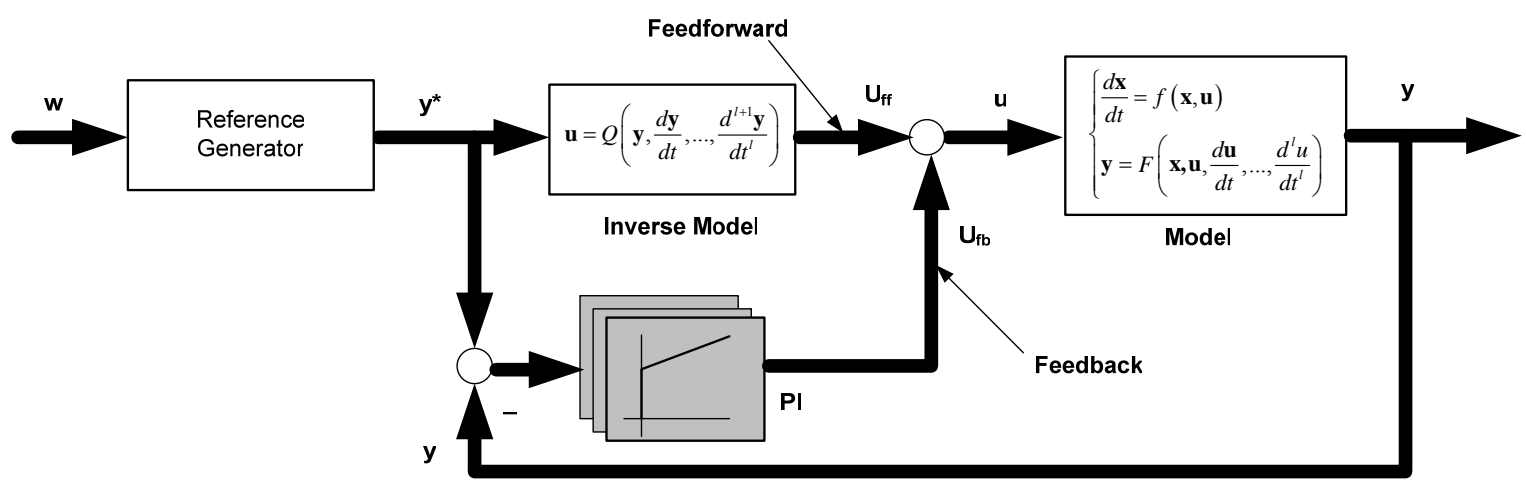

Figure 1. The general flatness-based control structure

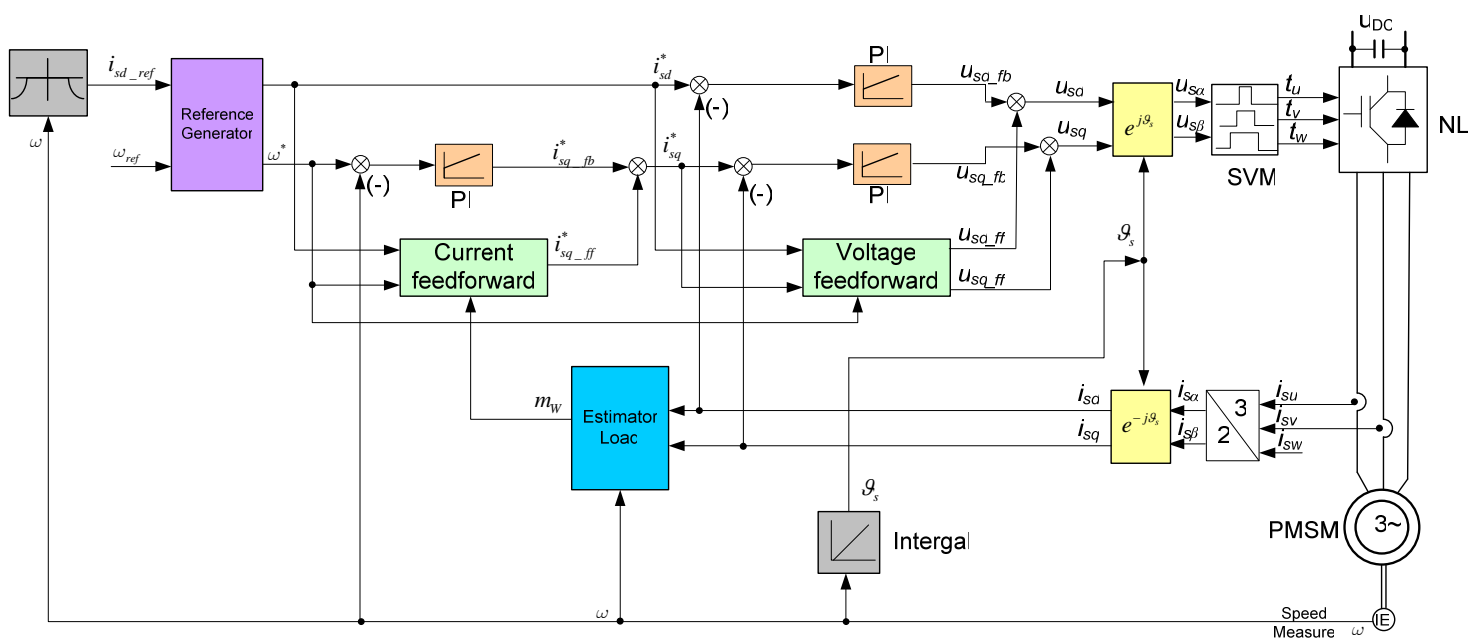

Figure 2. Cascaded control structure of flatness-based control of PMSM

where $T$ is sampling period and $\Xi(T)$ is the higherorder terms of the Taylor's series expansion which can be expressed as follows

$$
\begin{aligned}
& \Xi(T)=\frac{1}{2 !} x^{(2)}(k T) T^{2}+\ldots+\frac{1}{n !} x^{(n)}(k T) T^{n}+ \\
& +\frac{1}{(n+1) !} x^{(n+1)}(\zeta) T^{n+1}, \zeta \in(k T, k T+T) .
\end{aligned}
$$

As the sampling period in the advanced electric drive systems is very small, the higher-order terms in equation (6) can therefore be neglected. By substituting (1) into (5), the discrete-time current model of PMSM is obtained as

$$
\begin{aligned}
& x(k+1)=x(k)+T f(x(k))+T H(x(k)) u(k)+\Xi(T), \\
& y(k)=g(x(k)) .
\end{aligned}
$$

Equation (7) can be rewritten in the form of $\left\{\begin{array}{l}i_{s d}(k+1)=(1-c T) i_{s d}(k)+a T u_{s d}(k)+\frac{a}{b} T i_{s q}(k) \omega_{s}(k), \\ i_{s q}(k+1)=(1-d T) i_{s q}(k)+b T u_{s q}(k)-\frac{b}{a} T i_{s d}(k) \omega_{s}(k)-b T \psi_{p} \omega_{s}(k), \\ \vartheta_{s}(k+1)=\vartheta_{s}(k)+T \omega_{s}(k),\end{array}\right.$

where $a=\frac{1}{L_{s d}} ; b=\frac{1}{L_{s q}} ; c=\frac{1}{T_{s d}} ; d=\frac{1}{T_{s q}}$.

Note that the nonlinear characteristics in the current model of PMSM (8) is considered in terms of the products between the state variables (current components $\left.i_{s d}(k), i_{q}(k)\right)$ and input variable $\left(\omega_{s}(k)\right)$. Fom (8), by using the property of differential flatness whereas the trajectory inputs $u_{s d_{-} f f}(k)$ and $u_{s q_{-} f f}(k)$ can be directly determined from desired trajectory of flat outputs $i_{s d}^{*}(k), i_{s q}^{*}(k)$ and $\omega_{s}^{*}(k)$. Controllers for voltage feedforward block is proposed as 
$u_{s d_{-f f}}(k)=\frac{1}{a T}\left[i_{s d}^{*}(k+1)-(1-c T) i_{s d}^{*}(k)-\frac{a}{b} T i_{s q}^{*}(k) \omega_{s}^{*}(k)\right]$,

$u_{s q_{-f}}(k)=\frac{1}{a T}\left[i_{s q}^{*}(k+1)-(1-d T) i_{s q}^{*}(k)+\frac{b}{a} T i_{s d}^{*}(k) \omega_{s}^{*}(k)+b T \psi_{p} \omega_{s}^{*}(k)\right]$.

From (9), it can be seen that the coupling effects caused by decoupling current component $i_{s d}$ and $i_{s q}$ can be eliminated by $-\frac{a}{b} T i_{s q}^{*}(k) \omega_{s}^{*}(k)$ and $\frac{b}{a} T i_{s d}^{*}(k) \omega_{s}^{*}(k)$ terms.

Denote the winding time constants $T_{s d}=\frac{L_{s d}}{R_{s}}$ and $T_{s q}=\frac{L_{s q}}{R_{s}}$, the control parameters of feedback controller (PI controller) in the current loop is therefore determined as

$K_{p_{-} d}=\frac{2 L_{s d}}{\varepsilon_{d} T_{s d}}-R_{s}, K_{i_{-} d}=\frac{L_{s d}}{\varepsilon_{d}^{2} T_{s d}^{2}}$,
$K_{p_{-} q}=\frac{2 L_{s q}}{\varepsilon_{q} T_{s q}}-R_{s}, K_{i_{-} q}=\frac{L_{s q}}{\varepsilon_{q}^{2} T_{s q}^{2}}$,

where $\varepsilon_{d}, \varepsilon_{q}$ are positive scalars such that $\varepsilon_{d} T_{s d}>0, \varepsilon_{q} T_{s q}>0$.

Thus, the current controller is obtained as

$u_{s d_{-} f b}(k)=u_{s d_{-} f b}(k-1)+r_{0 i_{-} d}\left[i_{s d}^{*}(k)-i_{s d}(k)\right]+r_{1 i_{-} d}\left[i_{s d}^{*}(k-1)-i_{s d}(k-1)\right]$,

$u_{s q_{-} f b}(k)=u_{s q_{-} f b}(k-1)+r_{0 i_{-} q}\left[i_{s q}^{*}(k)-i_{s q}(k)\right]+r_{1 i_{-} q}\left[i_{s q}^{*}(k-1)-i_{s q}(k-1)\right]$,

where

$r_{0 i_{-} d}=K_{p_{-} d}+T_{s i} \cdot \frac{K_{i_{-} d}}{2} ; r_{0 i_{-} q}=K_{p_{-} q}+T_{s i} \cdot \frac{K_{i_{-} q}}{2}$,

$r_{1 i_{-} d}=T_{s i} \cdot \frac{K_{i_{-} d}}{2}-K_{p_{-} d} ; r_{1 i_{-} q}=T_{s i} \cdot \frac{K_{i_{-} q}}{2}-K_{p_{-} q}$, and $T_{s i}$ is the sampling time for current loop.

\subsubsection{Speed controller}

The motion equation of PMSM is considered as

$J \frac{d \omega}{d t}=\frac{3}{2} z_{p}\left[\psi_{p}+i_{s d}\left(L_{s d}-L_{s q}\right)\right] i_{s q}-m_{w}$.

Similarly, by adopting the discretization approximation (5), the discrete-time speed model of the PMSM is obtained as follows

$$
\begin{aligned}
& J \frac{1}{2 T}[3 \omega(k)-4 \omega(k-1)+\omega(k-2)]= \\
& =\frac{3}{2} z_{p}\left[\psi_{p}+i_{s d}(k)\left(L_{s d}-L_{s q}\right)\right] i_{s q}(k)-m_{W} .
\end{aligned}
$$

Based on the property of differential flatness, the controller of the current feedforward block is obtained as

$$
i_{s q_{-} f f}^{*}(k)=\frac{J \frac{1}{2 T}\left[3 \omega^{*}(k)-4 \omega^{*}(k-1)+\omega^{*}(k-2)\right]+m_{w}}{\frac{3}{2} z_{p}\left[\psi_{p}+i_{s d}^{*}(k)\left(L_{s d}-L_{s q}\right)\right]},
$$

and a feedback controller (PI controller)

$$
\begin{aligned}
& i_{s q_{-} f b}^{*}(k)=i_{s q_{-} f b}^{*}(k-1)+r_{0 \omega}\left[\omega^{*}(k)-\omega(k)\right]+ \\
& +r_{01}\left[\omega^{*}(k-1)-\omega(k-1)\right] .
\end{aligned}
$$

Finally, the speed controller can be obtained as

$$
\begin{aligned}
& i_{s q}^{*}(k)=i_{s q_{-} f f}^{*}(k)+i_{s q_{-} f b}^{*}(k) \\
& i_{s q}^{*}(k)=\frac{J \frac{1}{2 T}\left[3 \omega^{*}(k)-4 \omega^{*}(k-1)+\omega^{*}(k-2)\right]+m_{w}}{\frac{3}{2} z_{p}\left[\psi_{p}+i_{s d}^{*}(k)\left(L_{s d}-L_{s q}\right)\right]}
\end{aligned}
$$

$+i_{s q_{-} f b}^{*}(k-1)+r_{0 \omega}\left[\omega^{*}(k)-\omega(k)\right]+r_{01}\left[\omega^{*}(k-1)-\omega(k-1)\right]$,

where

$$
r_{0 \omega}=K_{p_{-} \omega}+T_{s \omega} \cdot \frac{K_{i_{-} \omega}}{2}
$$

$r_{1 \omega}=T_{s \omega} \cdot \frac{K_{i_{-} \omega}}{2}-K_{p_{-} \omega}$

and $K_{p_{-} \omega}, K_{i_{-} \omega}$ are respectively the proportional gain and integral gain of PI controller in the speed loop.

\section{Simulation results}

In this work, to verify the effectiveness of the proposed control strategy, we consider a standard PMSM with the following parameters as shown in Table 1.

Table 1. PMSM parameters

\section{Parameter}

Value

Rated output power $\quad P_{\text {rated }} \quad 0,4 \mathrm{~kW}$

Rated voltage $U_{\text {rated }} \quad 220 \mathrm{~V}$

Rated current

Instantaneous peak current

Rated torque

$1.27 \mathrm{Nm}$

Number of poles $p_{p}$ 
Rated rotated speed

$3000 \mathrm{rpm}$

Stator resistance $R_{\mathrm{s}}$

Equivalent inertia $J$

$0.000031 \mathrm{kgm}^{2}$

Stator inductance $L_{\mathrm{s}}$

$0.0065 \mathrm{H}$

Several scenarios were considered for simulation to assess the capability of the proposed controller. Here, the speed of PMSM will be controlled with a constant load and this load will be involved after $0.5 \mathrm{sec}$.

\section{Case 1:}

The reference speed is set at $157.1(\mathrm{rad} / \mathrm{s})$-forward speed and -157.1 ( $\mathrm{rad} / \mathrm{s})$-reverse speed and the motor will reverse rotation at $0.3 \mathrm{~s}$. The constant load involves at $0.5 \mathrm{sec}$.

Simulation results are shown in Fig 3-8.

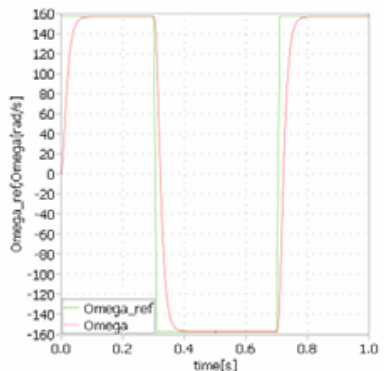

Figure 3. Speed Response

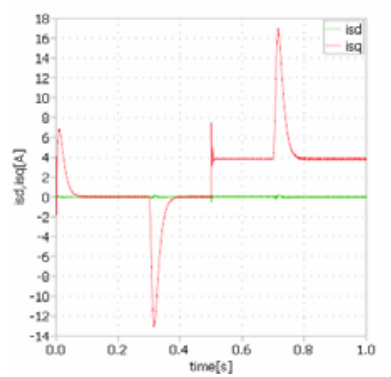

Figure 5. Current Response

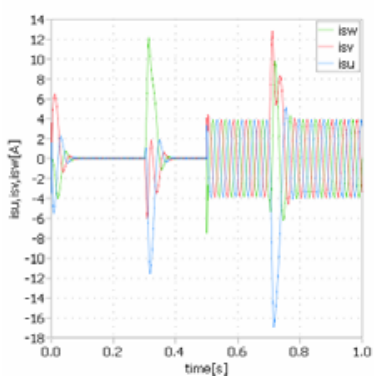

Figure 7. Response Three-Phase Current

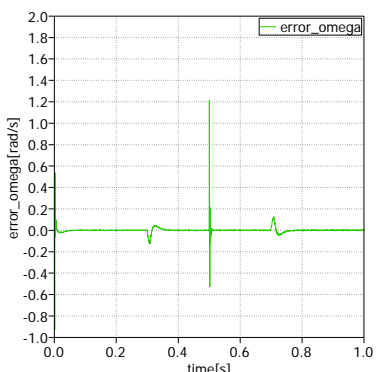

Figure 4. Speed Error

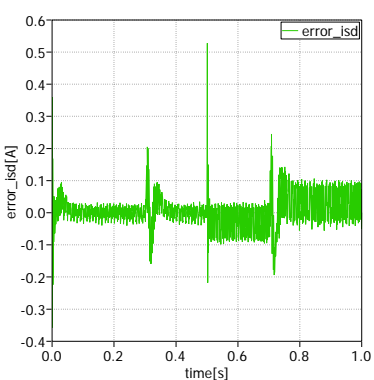

Figure 6. Error of $i_{\text {sd }}$

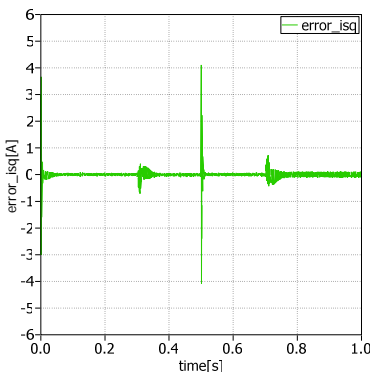

Figure 8. Error of $i_{\mathrm{sq}}$
Case 2:

The reference speed is set from $157.1(\mathrm{rad} / \mathrm{s})$ to 314.2 $(\mathrm{rad} / \mathrm{s})$ and the motor will reverse rotation at $0.7 \mathrm{~s}$. The constant load also involves at $0.5 \mathrm{sec}$.

Simulation results are given in Fig 9-14.

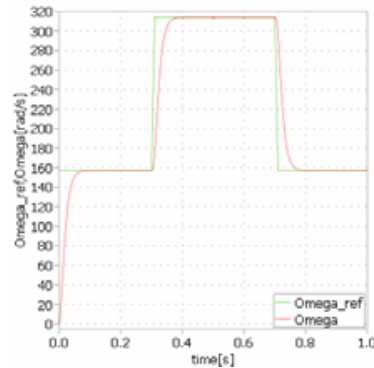

Figure 9. Speed Response

- Stator Current Response

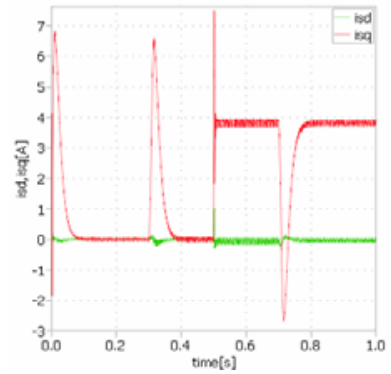

Figure 11. Response of component $i_{\text {sd }}$ and $i_{\text {sq }}$

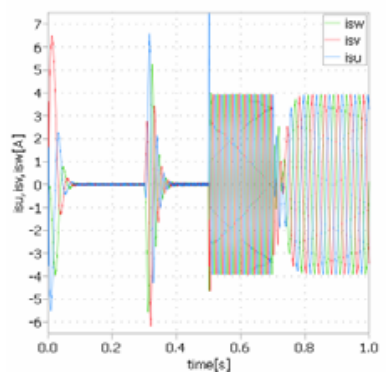

Figure 13. Response of Three-phase current

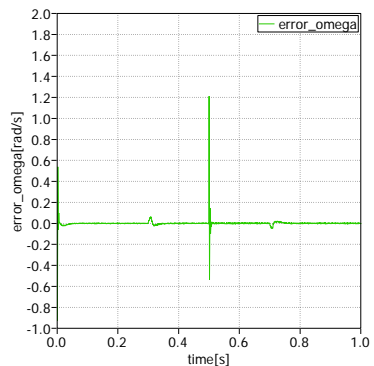

Figure 10. Speed Error

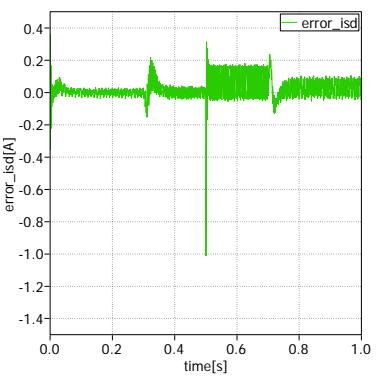

Figure 12. Error of $i_{s d}$

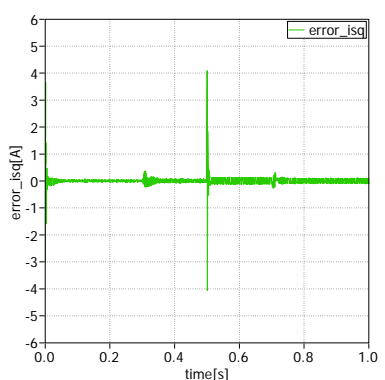

Figure 14. Error of $i_{\mathrm{sq}}$
It can be seen that the motor speed tracked the desired speed after $0.12 \mathrm{sec}$. The tracking error curves for both cases are depicted in Fig 2, 4, 6, 8, 10, 12, 14. It is apparent that the tracking performance of the proposed method in this study is good when the static errors including the speed and current errors converge into zero after $0.01 \mathrm{sec}$. It is also observed that the tracking errors are still within an acceptable level even when reversing the rotation and starting-up with a constant load. 


\section{Conclusion}

In this paper, the problem of nonlinear flatnessbased controller design of PMSM has been addressed. Based on Taylor series expansion and by using the property of differential flatness, two controllers of the current and speed loops are proposed to eliminate the static errors. Simulation results are provided to illustrate the feasibility of the proposed approach.

\section{References}

[1] E.C. Anene, U.O. Aliyu, J. Levine and G. K. Venayagamoorthy. Flatness-based feedback linearization of a synchronous machine model with static excitation and fast turbine valving. IEEE Power Engineering Society General Meeting, Tampa, FL, pp. 1-6, 2007.

[2] E. Delaleau and A.M. Stankovic, Flatness-based hierarchical control of the PM synchronous motor. Proceeding of the 2004 American Control Conference, Boston, Massachusetts, pp. 65-70, 2004.

[3] H.S. Ramirez. A passivity plus flatness controller for the permanent magnet stepper motor. Asian Journal of Control, vol. 2, no. 1, pp. 1-9, 2000.

[4] J. Dannehl and F.W. Fuchs. Flatness-based control of an Induction Machine Fed via Voltage Source Inverter-concept, Control Design and Performance Analysis. IEEE Industrial Electronics IECON 2006-32 ${ }^{\text {nd }}$ Annual Conference, Paris, pp. 51255130, 2006.

[5] J. Levine. Analysis and Control of Nonlinear Systems: A Flatness-based Approach. Springer, 2009.

[6] M. Fliess, J. Levine, P. Martin and P. Rouchon. Flatness and defect of nonlinear systems: introductory theory and examples. CAS internal report A-284, 1994.

[7] M. Fliess, J. Levine, P. Martin and P. Rouchon, Flatness and defect of non-linear systems: Introductory theory and examples. International Journal of Control, vol. 61, no. 6, pp. 1327-1361, 1995.

[8] P. Martin, R.M. Murray and P. Rouchon. Flatness systems, equivalence and trajectory generation, CDS Technical report, CDS 2003-008, 2003.

[9] N.P. Quang and J.A. Dittrich. Vector control of three-phase AC machines: system development in the practice. Springer, 2008.

[10] S. Thomson and F.W. Fuchs. Flatness based control of drive systems with resonant loads. The $36^{\text {th }}$ Annual Conference on IEEE Industrial
Electronics Society, Glendale, AZ, pp. 120-125, 2010.

[11] L. Fan and L. Zhang. Fuzzy based flatness control of an induction motor. Procedia Engineering, vol . 23, pp. 72-76, 2011.

[12] M. Fliess, J. Levine, P. Martin and P. Rouchon. On differentially flat nonlinear systems. In Proceedings of the IFAC-Symposium on Nonlinear Control Systems, Bordeaux, pp. 408-412, 1992.

[13] A. Houari, H. Renaudineau and J. Martin. Flatness based control of three phase inverter with output LC filter. IEEE Transactions on Industrial Electronics, pp. 2890- 2897, 2012.

[14] Z.H. Qu, Robust Control of Nonlinear Uncertain Systems, Wiley: New York, 1998.

[15] H. Thabet and M. Ayadi. Flatness-based trajectory generation for induction machine control. International Conference on Electrical Engineering and Software Applications, Hamamet, pp. 1-6, 2013.

[16] P.T. Thanh and N.P. Quang. Quasi-continuous implementation of structural nonlinear controller based on direct-decoupling for Permanent Magnet Synchronous Motor. IEEE International Conference on Control, Automation and Information Sciences, Nha Trang, Viet Nam, pp. 254-259, 2013. 